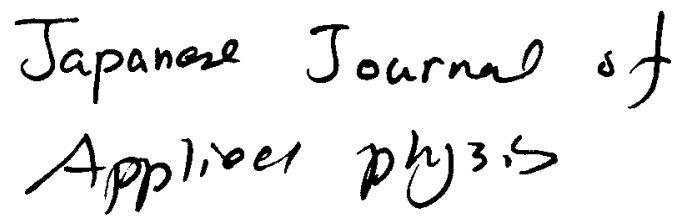

\title{
One Photon and Two Photon Process in Photo-Decomposition of Germanium Oxygen Deficient Centres
}

\author{
L. Dong, \\ Optoelectronics Research Centre, The University of Southampton, Southampton SO17 1BJ, UK \\ Tel: +44 1703 593163, Fax: +44 1703 593149, Email: LD@ORC.SOTON.AC.UK \\ and
}

V.N. Bagratashvili, S.I. Tsypina, Y.S. Zavorotny, A.O. Rybaltovskii, P.V. Chernov, S.S. Alimpiev and Y.O. Simanovskii

Laser Chemistry Research Centre, Pionerskava 2, Troitsk, Moscow Region 14092, Russia.

UV photon-induced transformation of germanium oxygen deficient centres (GODC) in germanium-doped silica glass have been studied using photocurrent measurements, absorption and fluorescence bleaching. It has been identified that the photocurrent are generated via a two photon effect. Evidence have been found suggesting that the UV photoninduced destruction of GODCs is achieved via two reaction pathways, a single photon pathway and a two photon pathway. The process is discussed.

KEYWORDS: glass photosensitivity, optical fibre gratings

\section{Introduction:}

There has been much debate on the fundamental mechanism of photosensitivity in germanium-doped silica optical fibres. The importance of the effect, which was discovered by K.O. Hill in 1978'), has been well demonstrated by the still rapidly increasing application areas that it has in fibre optics. Photo-induced index changes as high as $\sim 10^{-3}{ }^{2,3}$ ) and $\sim 0.01$ in $\mathrm{H}_{2}$ loaded fibres $^{4)}$ have enabled very strong gratings to be written conveniently into optical fibres to produce filters, wavelength defining reflectors, dispersion compensators, and sensors. The connection between the photosensitivity and the germanium-related oxygen deficient centre (GODC), first proposed by Hand and Russell ${ }^{\text {) }}$, is well recognised. Despite uncertainty on the exact microscopic structure of the GODC, the energy levels of the centre is well understood through spectroscopic studies of the centre. A diagram of such an energy level system is depicted in figure 1 , with singlet states marked by $\mathrm{S}$ and triplet states $\mathrm{T}$. Despite the fact there has been much work been done on the photodecomposition of GODCs, there is still work to be done for a thorough understanding the process. Potentially there are two possible reaction pathways. One is a direct two photon reaction producing a photoelectron in the conduction band. This process is resonantly enhanced by the GODC band at $\sim 240 \mathrm{~nm}$, therefore, is much stronger than that of conventional two photon effect for photon energy below the bandgap. Most of the photoelectrons will recombine with no net effect and some will be trapped at various sites to create a net change. The second reaction pathway is a single photon process involving the long lived triplet state and a near by trapping site, probably situated at the next co-ordination sphere. This is a local effect comparing with the long range effect of the two photon process and may well be in the form of the Pucker states as proposed by Sulimov'. The two situations are shown in figure 1 . Here
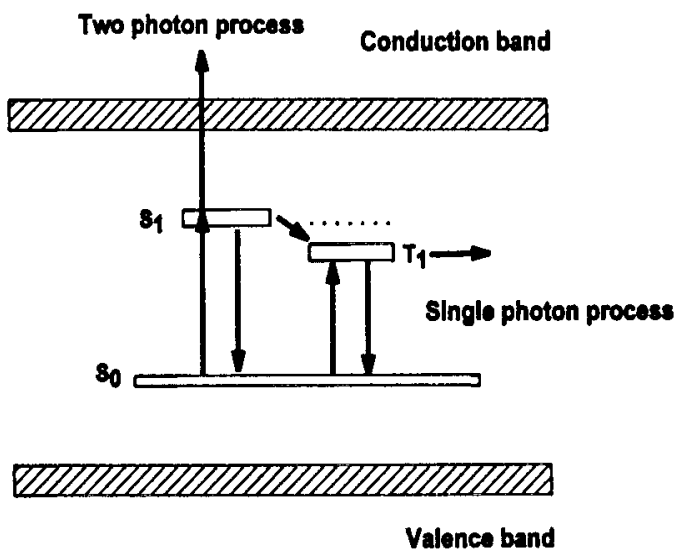

Figure 1. Energy level diagram of GODC and possible photodecomposition pathways. 
we report an experimental study on the reaction pathway of the GODCs, demonstrating that there are both single photon and two photon process existing under different conditions.

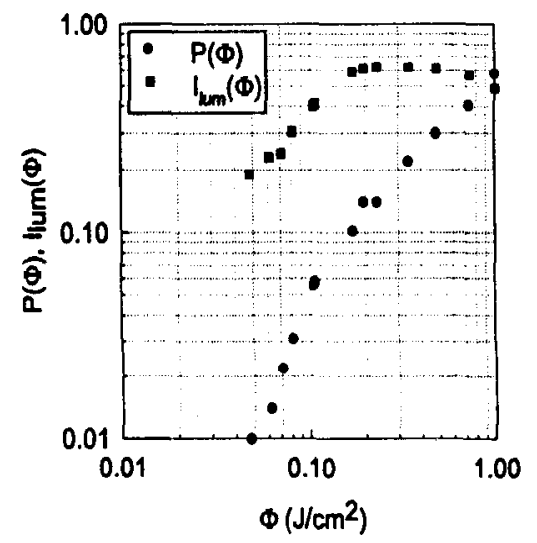

Figure 2 Fluence dependence of photo-current signal $P$ and photo-luminescence $I_{\text {lum }}$ for GODC in a germanium-doped silica sample.

\section{Experiments:}

All the samples used in the experiment were made by MCVD process and were well characterised in terms of germanium contents and GODC contents. The measurement of photocurrents was done in several different samples. A KrF excimer laser ( $248 \mathrm{~nm}, 25 \mathrm{~ns}$ pulse width) was used as the source. The samples with an area $\sim 5 \mathrm{~mm} \times 5 \mathrm{~mm}$ of polished faces were placed between two electrodes smaller than the sample to minimise background photocurrent caused by ionisation of air. The laser beam was parallel to the electrodes and carefully placed so as not to hit the electrodes. Simultaneously, the laser induced luminescence was collected into a monochromator, monitored by a photomultiplier and displayed on an oscilloscope. The temporal resolution of the our photocurrent measurement $(10 \mu \mathrm{s})$ was much longer than the lifetime of the free carriers $(0.01-1 \mathrm{~ns})$, the amplitude of the photo-current is proportional to the total number of displaced elementary charges, $\mathrm{P} \sim \delta \mathrm{Q}$. Rapid pulse to pulse decrease of photocurrent was observed. This is due to the screening effect from the produced internal field. The GODC centres remained more or less constant throughout as observed by the photo-luminescence. The decrease of photocurrent could be recovered by reversing the external field. Figure 2 gives the fluence dependence of photocurrent $P(\Phi)$ and photo luminescence $I_{\text {hum }}(\Phi)$ of GODC on sample Gl (OH 200 ppm, Al, Na $\sim 10$ ppm, Ge $100 \mathrm{ppm}$, thickness $14 \mathrm{~mm}) . \mathrm{P}(\Phi)$ increases with a slope of $\sim 2$ below $\sim 0.1 \mathrm{~J} / \mathrm{cm}^{2}$, above which a slope of $\sim 1$ was observed. For $I_{\text {lum }}(\Phi)$, it increases linearly with $\Phi$ below $0.2 \mathrm{~J} / \mathrm{cm}^{2}$ and then reaches a saturation before actually falling at higher fluence $\left(\Phi>0.5 \mathrm{~J} / \mathrm{cm}^{2}\right)$. The $\mathrm{P}(\Phi)$ slope of 2 at low intensity is a clear indication of a two photon effect via the $S_{1}$ or possibly $T_{1}$ states. The change of slope from 2 to 1 is due the depletion of the ground states $S_{0}$ population. This is evident from the luminescence measurement. The photocurrent is still a two photon effect despite the linear dependence at high pulse intensity.

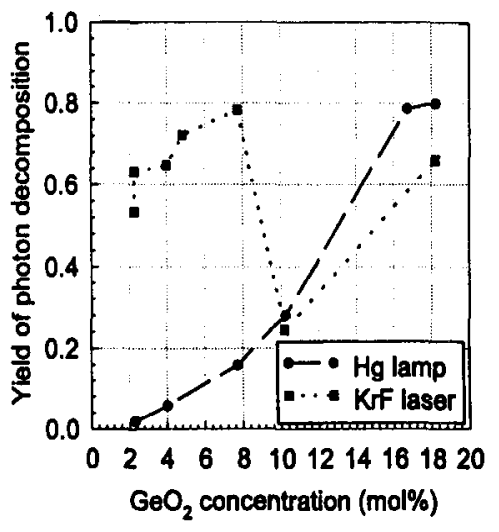

Figure 3 Germanium concentration dependence of the photoreaction yield with $\mathrm{Hg}$ lamp $\left(5 \mathrm{~mW} / \mathrm{cm}^{2}\right)$ and $\mathrm{KrF}$ excimer laser $\left(250 \mathrm{~mJ} / \mathrm{cm}^{2}\right)$ irradiation.

The yield of photo-reaction was also measured in a range of germanium-doped silica samples with different concentrations. Two types of exposures were used, one being a $\mathrm{Hg}$ lamp $\left(5 \mathrm{~mW} / \mathrm{cm}^{2}\right)$ for 10 minutes and the other being a $\mathrm{KrF}$ laser $\left(250 \mathrm{~mJ} / \mathrm{cm}^{2}\right)$ for 40 pulses. The results were shown in figure 3. For the exposure to Hg lamp, a monotonic increase of yield with 
germanium concentration was seen. This is an indication that the electron traps are some germanium related sites, increasing with germanium concentration. For the exposure to $\mathrm{KrF}$ Excimer laser, two regimes obviously exist. The transition seems to happen at $\sim 10 \mathrm{~mol} \%$. In each regime, the yield increases with germanium concentration, again due to an increase in the number of trapping sites. At low concentration, a higher yield is actually seen. We believe that the two photon reaction path way is dominate at low germanium concentration, due to the small likely hood of a nearby trapping sites to enable the single photon reaction. The photoelectrons generated in this case can access more stable trapping sites further away and therefore higher yield. At high germanium concentration $(>10 \mathrm{~mol} \%)$, the single photon process dominates due the availability of nearby trapping centres to react with the long lived triplet state. The low overall yield is due to the unstable nature of the centres. This effect seems to correlate well with some works done in low germanium doped fibres which can achieved $\sim 10^{-3}$ photo-induced index change much higher that was demonstrated in highly doped germanium fibres ${ }^{\eta}$.

To summarise, we have found that photocurrent is created through a two photon effect in germanium doped silica glass. Both single and two photon process can be involved in the decomposition of GODCs. At highly doped glass or at low intensity, single photon process dominates. In the lowly doped glass and at high intensity, a two photon process dominates.

\section{Acknowledgement:}

One of the authors, S.I. Tsypina, is would like to thank the Royal Society for the fellowship in support of the work.

\section{References:}

1. K.O. Hill, Y. Fujii, D.C. Johnson and B.S. Kawasaki: "Photosensitivity in optical fibre waveguides: application to reflection filter fabrication", Applied Physics Letters, 32(1978), pp.647-649.

2. D.L. Williams, B.J. Ainslie, J.R. Armitage, and R. Kashyap: "Enhanced photosensitivity in germania doped silica fibres for future optical network", 18th European Conference on Optical Communication, Berlin, 1992.
3. L. Dong, J.L. Cruz, L. Reekie, M.G. Xu and D.N. Payne: "Enhanced photosensitivity in tin-codoped germanosilicate optical fibres", IEEE Photonics Technology Letters, 9(1995), pp.1048-1050.

4. P.J. Lemaire, R.M. Atkins, V. Mizrahi and W.A. Reed: "High pressure $\mathrm{H}_{2}$ loading as a technique for achieving ultrahigh UV photosensitivity and thermal sensitivity in $\mathrm{GeO}_{2}$ doped optical fibres", Electronics Letters, 29(1993), pp.1191-1193.

5. D.P. Hand and P.St.J. Russell: "Photoinduced refractive index changes in germanosilicate fibres", Optics Letters, 15(1990), pp.102-104.

6. V.B. Sulimov, V.O. Sokolov, E.M. Dianov, and B. Poumellec" Photoinduced structural transformation in silica glass: mechanism for UV-written refractive index gratings?", Photosensitivity and Quadratic Nonlinearity in Glass Waveguides: Fundamental and Applications, OSA Tecjnical Digest Series, 22(1996), PD3-1-5.

7. H.G. Limberger, P.Y, Fonjallaz and R.P. Salathé: "Spectral characterisation of photoinduced high efficient Bragg gratings in standard telecommunication fibres", Electronics Letters, 29(1993), pp.47-49. 